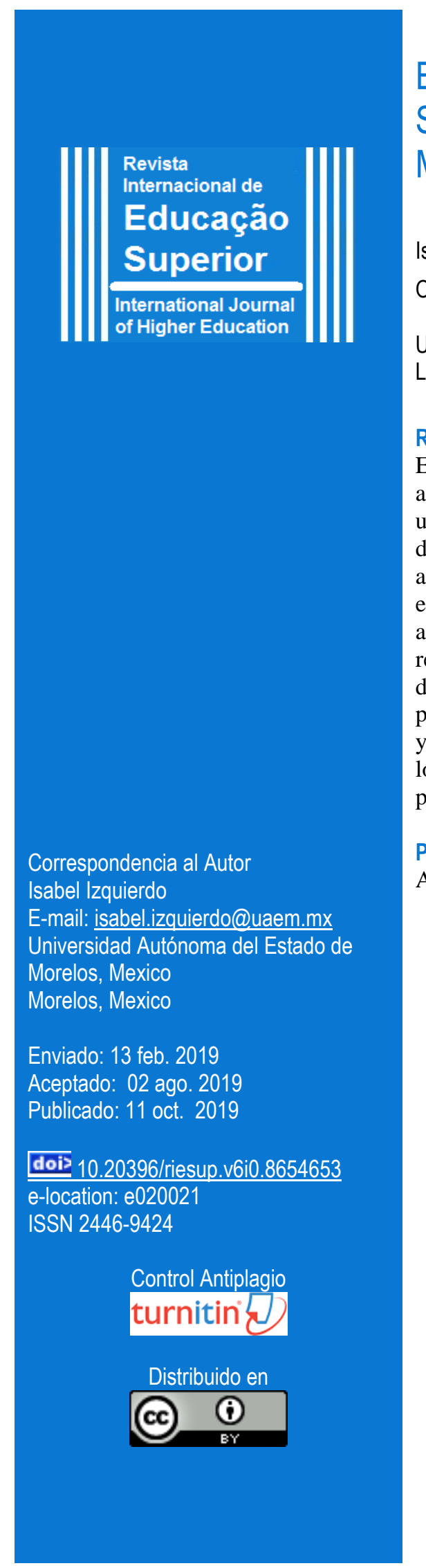

\title{
Experiencias de Migración de Académicas Sudamericanas en dos Universidades Públicas Mexicanas \\ Isabel Izquierdo1 ${ }^{(D)}$ http://orcid.org/0000-0002-3682-6862 \\ Olga Nelly Estrada2 ${ }^{2}$ http://orcid.org/0000-0002-3400-569X
}

Universidad Autónoma del Estado de Morelos ${ }^{1}$, Universidad Autónoma de Nuevo León ${ }^{2}$

\section{RESUMEN}

El objetivo del artículo es analizar las experiencias de migración de académicas sudamericanas, su llegada e incorporación en dos universidades mexicanas, una en el centro-sur y otra ubicada en el noreste del país. Partimos del supuesto que en su proceso migratorio, las académicas adquirieron una serie de experiencias que les permitió poner en práctica estrategias para transitar de situaciones de tensión a un aprendizaje en dicho proceso. Se utilizó el método biográfico, a través de relatos de vida, entrevistando a académicas que provienen, en su mayoría, de países de Sudamérica. Los hallazgos mostraron que las académicas pusieron en marcha estrategias de mimetización, creatividad, pragmatismo y de perseverancia, pese a las diferencias de desarrollo socioeconómico de los estados y del desarrollo en la investigación en las universidades públicas en donde laboran.

\section{PALABRAS CLAVE}

Académicas sudamericanas. Experiencias, México. 


\section{Migration Experiences of South American Women Faculty in Two Mexican Public Universities}

\section{ABSTRACT}

The aim of the article is to analyze the experiences of migration of South American women scholars, their arrival and incorporation in two Mexican universities, one in the center-south and another located in the northeast of the country. We assume that in their migratory process, the academics acquired a series of experiences that allowed them to implement strategies to move from stress situations to learning in that process. The biographical method was used, through life stories, interviewing scholars who mostly come from South American countries. The findings showed that scholars launched strategies of mimicry, creativity, pragmatism and perseverance, despite differences in socioeconomic development of states and research development in public universities where they work.

\section{KEYWORDS}

South American women scholars. Experiences. Mexico.

\section{Experiências De Migração de Acadêmicas Sul-americanas em Duas Universidades Públicas Mexicanas}

\section{RESUMO}

O objetivo do artigo é analisar as experiências de migração de acadêmicas sul-americanas, sua chegada e incorporação em duas universidades mexicanas, uma no centro-sul e outra localizada no nordeste do país. Assumimos que, em seu processo migratório, as acadêmicas adquiriram uma série de experiências que lhes permitiram implementar estratégias para passar de situações de estresse para a aprendizagem nesse processo. Utilizou-se o método biográfico, por meio de histórias de vida, entrevistando acadêmicas que vêm principalmente de países sul-americanos. Os achados mostraram que as acadêmicas lançaram estratégias de mimetismo, criatividade, pragmatismo e perseverança, apesar das diferenças no desenvolvimento socioeconômico dos estados e no desenvolvimento da pesquisa em universidades público onde trabalham.

\section{PALAVRAS-CHAVE}

Acadêmicas sul-americanas. Experiências. México. 


\section{Introdução}

La relevancia del fenómeno migratorio en el siglo XX a menudo fue enmarcada por las causas que generaron los desplazamientos, por las cifras totales de los flujos o por los países de origen y destino que participaron en él. Durante mucho tiempo, en el recuento de esos datos, se dejó de lado una parte central para analizar y entender dicho fenómeno de manera integral: las mujeres. Por lo menos hasta mediados de los sesenta, "el análisis de la migración femenina constituyó una ausencia recurrente en los estudios de población" (ARIZA, 2000, p. 39). Las primeras aproximaciones sobre las mujeres migrantes, las situaban dentro de "los grupos vulnerables desprotegidos y como receptoras pasivas del desarrollo, generalmente fueron descritas como la esposa o madre del hombre iniciador de la migración" (GREGORIO, 1998, p. 24).

Los aportes que mostraron la importancia del trabajo de las mujeres en las migraciones internacionales y el desarrollo, se dieron más tarde, a través del énfasis en el rol productivo femenino y de la igualdad de oportunidades en las relaciones entre las mujeres y los hombres en el mercado (AJAMIL, 1999 citado en DE VILLOTA, 1999); parte de esas reflexiones contribuyeron para contradecir la idea de que sólo los hombres tenían aportaciones en los espacios públicos. Fue hasta 1980 cuando se reconoció plenamente a las mujeres migrantes a través de su trabajo y su capacidad de organización social (GREGORIO, 1998; DE VILLOTA, 1999; PARELLA, 2003). Fue desde esa década que se inició una "nueva etapa en los estudios sobre la mujer migrante desde una perspectiva de género" (WOO, 1995, p. 143).

En los noventa, los amplios grupos de mujeres en las migraciones internacionales fueron identificados como la feminización de los flujos (CASTLES y MILLER, 1993), para dar cuenta de su creciente participación. Una década después, algunas organizaciones (ONU, 2006) señalarían que las mujeres "representan a 56 millones de un total de 118 millones de migrantes, una razón aproximada de 91 mujeres por 100 hombres" (PNUD, 2000, citado en LIPSZYC, 2004, p. 7).

La visibilización de las mujeres en las migraciones calificadas se dio hasta años recientes a nivel internacional y regional, cuando se empezaron a analizar las migraciones de mujeres académicas, científicas, empresarias y profesionales (por ejemplo, véase a: STANG, 2005; KOFMAN y RAGHURAM, 2005; IREDALE, 2005; SKACHKOVA, 2007; RIAÑO y BAGHDADI, 2007). Especialmente esos estudios documentaron las migraciones de las mujeres calificadas del Sur hacia el Norte.

Al acercamiento que presentamos sobre las mujeres migrantes, lo preceden otros estudios que se han desarrollado desde Latinoamérica, algunos de los cuales se centran en las profesoras, empresarias y científicas (MEYER; SALGADO, 2002; STANG, 2005; RIAÑO, 2007; ORTIZ; MENDOZA, 2007; HOPENHAYM, 2009; IZQUIERDO, 2010; ESTRADA; IZQUIERDO, 2011; FLORES, 2011; ROMO, 2015; IZQUIERDO y GUZMÁN, 2016) a los cuales nos hemos aproximado con el fin de recuperar y analizar la experiencia de las

\begin{tabular}{|l|l|l|l|l|l|}
\hline (C) Rev. Inter. Educ. Sup. & Campinas, SP & v.6 & $1-20$ & e020021 & 2020 \\
\hline
\end{tabular}


académicas migrantes desde los espacios académicos del Sur global, particularmente nuestra indagación se centró en México, a partir de un trabajo de campo que se desarrolló en dos universidades públicas en el interior de la República mexicana.

El filósofo español exiliado en el país y que trabajó en la Universidad Nacional Autónoma de México (UNAM), Adolfo Sánchez Vázquez, decía que "lo decisivo no es estar -acá o allá- sino cómo se está" en el exilio (SÁNCHEZ, 1997, p. 47). En esta investigación de corte exploratoria, se intentó conocer "cómo se está" en la movilidad y en la migración de las académicas provenientes de países sudamericanos (en su mayoría) que viven y trabajan en México; interesó conocer ¿Por qué se mueven y migran hacia espacios académicos del Sur global? ¿Cómo fueron sus experiencias en esos espacios particulares? Y ¿Qué estrategias pusieron en práctica y por qué? Partimos del supuesto que en su proceso migratorio, las académicas adquirieron una serie de experiencias que les permitió poner en marcha estrategias para transitar de situaciones de tensión a un aprendizaje de saber estar en contextos migratorios.

Desde el campo educativo, la experiencia se entiende como "eso que me pasa" (LARROSA, 2006, p. 88); pero la experiencia también supone un acontecimiento particular, específico, no todo es experiencia, debe ser algo "no previsto", algo novedoso para el sujeto. Es un acontecimiento que no depende del sujeto, es "exterior" al sujeto; es un acontecimiento que irrumpe, que sucede "en mí”, no "ante mí o frente a mí” (p. 89). Según Larrosa (2006), la experiencia se va a suscitar de forma única en cada sujeto, pero para que esto sea posible, es preciso que el sujeto realice una introspección de lo que le acontece, representando la tensión (o tensiones) que le produce determinada situación y cómo eso puede perturbar su tranquilidad y será mediante la puesta en marcha de acciones o estrategias que el sujeto podría resolver o gestionar las tensiones que se le presentan. Las tensiones fueron entendidas en este estudio, de acuerdo a Dubar (2002), como puntos de crisis que no evitan ni frenan a los sujetos para la acción, sino que la pueden promover. Las acciones o estrategias son definidas como "un ensamble de acciones coordinadas para obtener una victoria, en el nivel interaccional y que es dinámica" (KASTERSZEIN, 1999, p. 30). El fin último de poner en práctica una estrategia es el reconocimiento del sujeto, de su existencia específica en una sociedad, institución o grupo, en este caso nos referimos al contexto de dos universidades públicas en México.

\section{Metodología}

En la investigación se utilizó el método biográfico, a través del relato de vida (BERTAUX, 1993). Se realizaron diez entrevistas semiestructuradas (VALLES, 2002) a académicas adscritas en dos universidades públicas mexicanas (centro-sur y noreste), se indagaron particularmente tres ejes de análisis en las entrevistas: motivos de la movilidad y migración, llegada y trabajo académico (tensiones) en México y las acciones que pusieron en práctica para resolver las tensiones. Se solicitó una entrevista a las académicas de ambas universidades (11 y 8 respectivamente) provenientes de países latinoamericanos sin 
embargo, no tuvimos respuesta de todas ellas y al final, el estudio se llevó a cabo solo con las investigadoras que respondieron a la solicitud, la mayoría de ellas provenientes de países sudamericanos: Brasil (1), Argentina (2), Colombia (4), Cuba (2) y Panamá (1), de áreas de las ciencias exactas e ingeniería, ciencias sociales, humanidades y educación, ciencias naturales, ciencias de la salud y del comportamiento.

Tabla 1. Las académicas participantes en el estudio

\begin{tabular}{|c|c|c|c|c|c|}
\hline Participante & Edad & $\begin{array}{l}\text { País de } \\
\text { origen }\end{array}$ & SNI & $\begin{array}{c}\text { Nombre del } \\
\text { doctorado obtenido }\end{array}$ & $\begin{array}{c}\text { Año de llegada } \\
\text { a México }\end{array}$ \\
\hline 1-pan-unor & $\begin{array}{l}46 \\
\text { años }\end{array}$ & Panamá & $\overline{1}$ & $\begin{array}{l}\text { Doctorado en } \\
\text { Ingeniería en } \\
\text { Materiales }\end{array}$ & 1989 \\
\hline 2-col-unor & $\begin{array}{c}51 \\
\text { años }\end{array}$ & Colombia & 1 & $\begin{array}{l}\text { Doctorado en } \\
\text { Derecho }\end{array}$ & 2001 \\
\hline 3-bra-unor & $\begin{array}{c}72 \\
\text { años }\end{array}$ & Brasil & 1 & $\begin{array}{l}\text { Doctorado en } \\
\text { Ciencias Políticas }\end{array}$ & 1981 \\
\hline 4-arg-unor & $\begin{array}{c}68 \\
\text { años }\end{array}$ & Argentina & 3 & $\begin{array}{l}\text { Doctorado en } \\
\text { Ciencias Químicas }\end{array}$ & 1981 \\
\hline 5-cub-unor & $\begin{array}{l}67 \\
\text { años }\end{array}$ & Cuba & 1 & $\begin{array}{l}\text { Doctorado en } \\
\text { Ciencias Químicas }\end{array}$ & 1993 \\
\hline 1-cub-ucsur & $\begin{array}{l}43 \\
\text { años }\end{array}$ & Cuba & no & $\begin{array}{l}\text { Doctorado en } \\
\text { Psicología }\end{array}$ & 1999 \\
\hline 2-col-ucsur & $\begin{array}{l}55 \\
\text { años }\end{array}$ & Colombia & 1 & $\begin{array}{l}\text { Doctorado en } \\
\text { Educación }\end{array}$ & 1982 \\
\hline 3-arg-ucsur & $\begin{array}{l}42 \\
\text { años }\end{array}$ & Argentina & no & $\begin{array}{l}\text { Doctorado en } \\
\text { Ecología }\end{array}$ & 2004 \\
\hline 4-col1-ucsur & $\begin{array}{l}48 \\
\text { años }\end{array}$ & Colombia & 1 & $\begin{array}{l}\text { Doctorado en } \\
\text { Medicina }\end{array}$ & 2002 \\
\hline 5-col2-ucsur & $\begin{array}{l}42 \\
\text { años }\end{array}$ & Colombia & $\mathrm{C}$ & $\begin{array}{l}\text { Doctorado en } \\
\text { Botánica }\end{array}$ & 2003 \\
\hline
\end{tabular}

Fuente: elaboración propia con base en el trabajo de campo realizado

Algunos elementos de la heterogeneidad de la movilidad y migración, así como de la escolaridad y especialización en la ciencia de las participantes en el estudio, se mostró en la Tabla 1. Las académicas llegaron principalmente al país en los ochenta y en el dos mil. En la década de los ochenta en México se impulsó una de las políticas más importantes para la retención de científicos/as: el Sistema Nacional de Investigadores (SNI) ${ }^{1}$. Llama la atención que en comparación con este grupo de académicas, existen otros como los provenientes de la ex Unión Soviética, Alemania y Francia, por mencionar algunos, quienes llegaron principalmente en la década de los noventa (IZQUIERDO, 2011), justo cuando el gobierno mexicano puso en marcha diferentes programas para la atracción de personal calificado a

\footnotetext{
${ }^{1}$ El SNI fue creado "para reconocer la labor de las personas dedicadas a producir conocimiento científico y tecnología. El reconocimiento se otorga a través de la evaluación por pares y consiste en otorgar el nombramiento de investigador nacional. Esta distinción simboliza la calidad y prestigio de las contribuciones científicas. En paralelo al nombramiento, se otorgan estímulos económicos cuyo monto varía con el nivel asignado" (Consejo Nacional de Ciencia y Tecnología, www.conacyt.mx).
} 
nível internacional y desarrolló acciones, en conjunto con las diferentes autoridades de las universidades públicas, para el impulso de la internacionalización de la Educación Superior. Acciones que se centraron principalmente en la movilidad estudiantil y del profesorado, así como intercambios y estancias de investigación y de formación en la ciencia. Todo lo anterior conformó un contexto de oportunidades para que algunas de las académicas del estudio llegarán al país también en la década del dos mil.

\section{Las Universidades Mexicanas ¿Receptoras de Inmigración Internacional Calificada? Indagación en dos Universidades Públicas}

México ha sido un país receptor de profesionistas exiliados e inmigrantes. En el tema que nos ocupa, las primeras inmigraciones datan de 1826 y provenían de la llamada República Centroamericana de Guatemala (MEYER y SALGADO, 2002). La historia más reciente de esas movilidades hacia el país abarcó desde 1940 hasta 1980, con los exilios provenientes de Perú, República Dominicana, Haití, Honduras, Bolivia, Costa Rica, Cuba (1940), Colombia, Brasil (1960), Chile, Argentina, Uruguay, Nicaragua (1970) y Guatemala (1980). Estas últimas movilizaciones humanas se caracterizaron por la persecución política de los gobiernos dictatoriales que se instalaron en América Latina y el Caribe en esas décadas (MEYER y SALGADO, 2002), con la ayuda de Estados Unidos y de los nacionales de esos países.

De todos esos grupos, no se cuentan con investigaciones tan amplias, como fue el caso del exilio español (1930-1940) que muestren estudios sobre profesionistas y de personal en la academia y en la ciencia que llegaron a tierras mexicanas y sus respectivas experiencias. Los estudios que hemos identificado hasta el momento en México, son de corte histórico y tienen que ver principalmente con el exilio y la migración proveniente de Chile, Argentina, Colombia y Cuba. En ese sentido coincidimos con Martínez-Assad (2008) que si bien todos los grupos de exiliados e inmigrantes que han llegado al país han influido en la construcción de la "nación mexicana" y de las instituciones educativas, como en el caso de las universidades públicas, todavía no se han estudiado con profundidad (p. 23) y desde los espacios de investigación y docencia en las universidades de recepción.

En décadas recientes el estudio sobre el profesorado y académicos inmigrantes en México cobró relevancia y fue desde los noventa y en el dos mil, cuando se mostró la participación de estos grupos a través de casos de estudio, así como de la puesta en marcha de programas específicos nacionales y en relación con agencias de cooperación en el marco de dos de las dimensiones de la internacionalización universitaria que identifica Oregioni (2017): educación e investigación. La primera a través de programas de "movilidad estudiantil" y la segunda, por "diásporas científicas" (p. 118), cuyas acciones se concretaron, en el caso de México, a través del Programa Internacional de Jóvenes Doctores en los noventas; el Subprograma de Cátedras Patrimoniales de Excelencia que se inauguró también en los noventa y se mantuvo hasta el dos mil dos y el Programa de Cátedras CONACYT para 
Jóvenes Investigadores (IZQUIERDO, 2010; CASTAÑOS, 2011; DELGADO, 2014; MENDOZA, STANISCIA; ORTÍZ, 2016; PEDONE; IZQUIERDO, 2018).

En los casos que presentamos, varias de las académicas entrevistadas llegaron al país por movilidad estudiantil y en busca de trabajo. De las diez profesoras investigadoras, 4 llegaron a México en la década de los ochenta, 2 en los noventa y 4 en el dos mil. Algunos de los elementos que señalamos a continuación sobre cada estado, uno en el centro-sur y otro ubicado en el noreste de la República mexicana al que llegaron, así como de cada universidad en donde se incorporaron laboralmente, muestran que las académicas arribaron a contextos e instituciones distintas, pero con experiencias de incorporación y tensiones en el trabajo académico, en varios puntos, coincidentes.

Con respecto a la población nacida en el extranjero residente en México, ninguno de los dos estados, el que se ubica en el noreste y el del centro-sur, figura en los cinco primeros lugares con ese tipo de población. En esos lugares se encuentran, según datos del Consejo Nacional de Población (CONAPO) del 2015, la Ciudad de México (13.5\%), Baja California (12.7\%), Jalisco (10.9\%), Chihuahua (7.5\%) y Tamaulipas (6.05\%). El lugar ocho lo ocupa el estado del noreste, con el $3.8 \%$ y el del centro-sur con el $0.8 \%$. Si bien a nivel nacional, la mayor población extranjera internacional que reside en el país son provenientes de Estados Unidos con un $69.7 \%$, seguido muy por debajo de España con un $4.2 \%$, se tienen registros que a nivel regional, algunos países de Centroamérica y Sudamérica también participan en esa población, entre ellos se encuentra Guatemala (4.9\%), Argentina (1.3\%) y Colombia $(1.2 \%)$, además de la población cubana que se ubica como la sexta población nacida en el extranjero residente en el país.

Las académicas llegaron a vivir a dos estados de la República muy diferentes entre sí. El estado ubicado en el noreste alberga 4, 653, 458 millones de habitantes, de los cuales 2, 333,273 son mujeres y 2, 320,185 son hombres; dicho estado es conocido como el centro comercial e industrial más importante del país, integrando más de 200 grupos industriales, siendo la industria manufacturera una de las más consolidadas, concentrando el 7. 5\% del PIB nacional (seguido de la Ciudad de México y del Estado de México). Por su parte, el estado localizado en el centro-sur tradicionalmente ha sido agricultor, en los sesenta y setenta fue uno de los principales productores y exportadores de caña de azúcar y arroz; según el Instituto Nacional de Estadística y Geografía (INEGI) ese estado cuenta con 1,777, 227 millones de habitantes $(918,639$ mujeres y 858,588 hombres) y concentra el $2.1 \%$ del PIB nacional.

Ambos estados también mantienen diferencias con respecto al desarrollo en el ámbito educativo. El estado situado en el noreste tiene un grado promedio de escolaridad de 9.8 años, es decir, que la mayoría de las personas de 15 años y más, tiene prácticamente el primer grado de educación media superior terminado. Este indicador está por encima del 8.6 nacional y la entidad ocupa la segunda posición respecto a los 32 estados que integran a la República mexicana, en este sentido el estado ubicado en el noreste es una de las entidades con más avances educativos del país (ESTRADA, 2012). El estado que se ubica en la zona centro-sur del país en cambio, cuenta con un grado promedio de su población de 15 y más años, de 8.9,

\begin{tabular}{l|l|l|l|l|l}
\hline C) Rev. Inter. Educ. Sup. & Campinas, SP & v.6 & $1-20$ & $\mathrm{e} 020021$ & 2020 \\
\hline
\end{tabular}


lo que significa que, según los datos recabados por el INEGI, dicha población cuenta con un nivel de educación básica (en México dicho nivel está conformado por la primaria, seis años, y secundaria, tres años), un nivel educativo abajo del estado del noreste.

Las universidades en donde se incorporaron laboralmente las académicas son iguales en el sentido de que ambas provienen del mismo subsistema de educación pública y, según lo estipulado en el portal de Internet de la Secretaría de Educación Pública (SEP), fueron "creadas por decreto de los congresos locales, bajo la figura jurídica de organismos públicos descentralizados. Estas instituciones estatales desarrollan las funciones de docencia, generación y aplicación innovadora del conocimiento, así como de extensión y difusión de la cultura" sin embargo, también entre ellas hay diferencias. Mientras la universidad del noreste fue creada en 1933, la del centro-sur se fundó dos décadas después, en 1953, eso marcó un desarrollo distinto entre ambas, especialmente en la investigación. Fue en la década de los ochenta cuando se institucionalizó el trabajo de investigación científica en el caso de la primera (ESTRADA, 2012), y en la segunda ese proceso se dio una década después, en los noventa. Ese fue uno de los elementos que posibilitó el crecimiento y desarrollo de la investigación y de la atracción (y posterior contratación) de investigadores/as nacidos/as fuera de México (y nacionales). Uno de los indicadores que puede mostrar lo anterior, por ejemplo, es el número de personal de Profesores/as Investigadores/as de Tiempo Completo (PITC) que forma parte del Sistema Nacional de Investigadores (SNI) del Consejo Nacional de Ciencia y Tecnología (CONACyT). Actualmente, la universidad del noreste cuenta con un profesorado de tiempo completo de 3,358, de esos registros 696 pertenecen al SNI, 255 son mujeres y 441 hombres. La universidad del centro-sur cuenta con 503 PITC, de esos, 279 (113 mujeres y 166 hombres) integran el SNI.

Los/as PITC nacidos/as en el extranjero que se han incorporado a ambas universidades provienen de un nutrido grupo de países, entre los que destacan por sus números Cuba y Colombia, en ese orden; la mayoría de las y los PITC se ubicaron, en primer lugar, en ciencias exactas, seguido, muy por debajo de Humanidades y Ciencias Sociales, llama la atención que, en general, hay más hombres que mujeres, pero en un análisis específico por áreas de conocimiento y por universidades, se percibe que se integran más mujeres en esas últimas áreas para el caso de la universidad del centro-sur, pero no sucede así en el caso de la del noreste para ninguna de las áreas de conocimiento, Tabla 2.

Tabla 2. PITC nacidos/as en el extranjero adscritos/as a dos universidades públicas

\begin{tabular}{|c|c|c|c|c|}
\hline Áreas de conocimiento & País & Mujeres & Hombres & Total \\
\hline \multicolumn{5}{|c|}{ Universidad del centro-sur } \\
\hline Ciencias Agropecuarias & --- & 0 & 0 & 0 \\
\hline Ciencias de la Salud y el & 1 Brasil & 1 & 0 & 1 \\
\hline \multirow{3}{*}{ Comportamiento } & Canadá & 0 & 1 & 1 \\
\hline & Colombia & 1 & 0 & 1 \\
\hline & Cuba & 1 & 0 & 1 \\
\hline \multirow[t]{4}{*}{ Ciencias Exactas e Ingeniería } & Alemania & 0 & 3 & 3 \\
\hline & Argentina & 0 & 1 & 1 \\
\hline & Colombia & 1 & 2 & 3 \\
\hline & Cuba & 0 & 3 & 3 \\
\hline (C) Rev. Inter. Educ. Sup. & Campinas, SP & $1-20$ & $\mathrm{e} 020021$ & 2020 \\
\hline
\end{tabular}


Ciencias Naturales

Ciencias Sociales
Administrativas

Humanidades y Educación

s/d

Total

Ciencias Agropecuarias

Ciencias de la Salud y Comportamiento

Ciencias Exactas e Ingeniería

Ciencias Naturales

Ciencias Sociales

Administrativas

\begin{tabular}{lllll} 
& Alemania & 1 & 0 & 1 \\
& India & 0 & 1 & 1 \\
& España & 0 & 3 & 3 \\
& Argentina & 0 & 1 & 1 \\
& Venezuela & 0 & 1 & 1 \\
& Italia & 0 & 1 & 2 \\
& España & 0 & 2 & 1 \\
& Argentina & 0 & 1 & 0 \\
\hline Tumanidades y Educación & El Salvador & 1 & 0 & 2 \\
\hline & Cuba & 1 & 1 & 41 \\
\hline
\end{tabular}

Fuente: elaboración propia con base en los datos obtenidos en ambas universidades.

$\begin{array}{llll}\text { Francia } & 0 & 1 & 1 \\ \text { India } & 1 & 0 & 1 \\ \text { Marruecos } & 0 & 1 & 1 \\ \text { Rusia } & 4 & 4 & 8 \\ \text { Ucrania } & 0 & 1 & 1 \\ \text { Vietnam } & 0 & 1 & 1 \\ \text { Argentina } & 1 & 0 & 1 \\ \text { Brasil } & 0 & 1 & 1 \\ \text { Colombia } & 1 & 0 & 1 \\ \text { Colombia } & 2 & 0 & 2 \\ \text { Croacia } & 1 & 0 & 1 \\ \text { Cuba } & 1 & 0 & 1 \\ \text { España } & 2 & 1 & 3 \\ \text { Inglaterra } & 1 & 0 & 1 \\ \text { Argentina } & 1 & 0 & 1 \\ \text { Colombia } & 1 & 2 & 3 \\ \text { España } & 3 & 1 & 4 \\ \text { Francia } & 1 & 1 & 2 \\ \text { Guatemala } & 0 & 1 & 1 \\ \text { Italia } & 1 & 0 & 1 \\ \text { s/d } & 1 & 5 & 6 \\ & 26 & 30 & 56\end{array}$

Universidad del noreste

Irán $\quad 0 \quad 1 \quad 1$

$\begin{array}{llll}\text { Colombia } & 0 & 1 & 1\end{array}$

$\begin{array}{llll}\text { Rusia } & 2 & 3 & 5\end{array}$

$\begin{array}{llll}\text { España } & 1 & 2 & 3\end{array}$

Finlandia $\quad 1 \quad 00$

Alemania $\quad 0 \quad 1 \quad 1$

$\begin{array}{llll}\text { Colombia } & 0 & 1 & 1\end{array}$

$\begin{array}{llll}\text { Francia } & 0 & 1 & 1\end{array}$

Panamá $\quad 1 \quad 001$

$\begin{array}{llll}\text { Cuba } & 2 & 0 & 2\end{array}$

$\begin{array}{llll}\text { Colombia } & 1 & 0 & 1\end{array}$

$\begin{array}{lll}\text { India } & 0 & 2\end{array}$

$\begin{array}{llll}\text { Bolivia } & 0 & 1 & 1\end{array}$

Argentina $\quad 1 \quad 001$

$\begin{array}{llll}\text { Cuba } & 1 & 0 & 1\end{array}$

$\begin{array}{lll}\text { Rusia } & 0 & 1\end{array}$

Venezuela $\quad 0 \quad 1 \quad 1$

$\begin{array}{llll}\text { España } & 0 & 1 & 1\end{array}$

y Inglaterra $\quad 1 \quad 001$


Si bien todo lo que hemos presentado, nos muestra algunas de las diferencias del contexto socio-histórico en cada uno de los estados en donde las académicas llegaron a vivir y a laborar, fue en la parte de la indagación en su incorporación laboral y de su trabajo académico en las dos universidades públicas mexicanas, en donde se esbozaron ciertas similitudes con respecto a sus experiencias en dichos ámbitos, así como de las estrategias que pusieron en marcha.

\title{
Experiencias en el Proceso de Migración de las Académicas: Entre Tensiones y Estrategias
}

La mayoría de las académicas tomaron la decisión de emigrar de sus países de origen para continuar con sus estudios de licenciatura y de posgrado. En algunos casos, el motivo fue para trabajar, dado que ellas ya habían obtenido el doctorado y llegaron al país para incorporarse como profesoras investigadoras en la universidad del noreste. En el caso de la universidad del centro-sur, todas las académicas llegaron a México por razón de estudios, especialmente quienes venían a realizar posgrados porque contaban con el respaldo de una beca que posibilitó sus movilidades, en algunos casos tanto para ellas como para sus compañeros de vida, otorgadas por el Consejo Nacional de Ciencia y Tecnología (CONACyT) y en un caso, por el Banco Interamericano de Desarrollo:

\begin{abstract}
Cuando llegué a México me vine con una beca que me gané junto con mi esposo para estudiar una maestría y después, el doctorado, nadie nos daba beca, en aquel tiempo buscamos en Perú, en Ecuador, pero no nos dieron beca, fue en México donde encontramos esta posibilidad de estar becados los dos, nos fue muy bien con las becas, nadie en ese tiempo te daba becas solo México (3-BRA-UNOR, 2017).

Un día llega el que en ese entonces era mi director en el trabajo y me dice "es su doctorado, esto está hecho para usted, pero la convocatoria cierra en 3 días". Era una convocatoria del Banco Interamericano de Desarrollo, ofrecía tres becas a toda Latinoamérica para el doctorado en México. Metí mis papeles, habían dicho que los resultados salían como en 2 meses, a los 2 meses no tuve ninguna noticia, entonces dije "no salí", pero mi director me dice "usted por qué es tan desconfiada, usted por qué no confía ni en usted, ni en sus capacidades, yo a usted ya la veo en México". Ya íbamos a cumplir los tres meses y me llega una carta diciendo que había sido preseleccionada, junto con un fulano, y que los dos íbamos a competir por Colombia por una de las becas. Tú no sabes lo que fue, eso a mí me dio autoconfianza y, luego, al saber que me gané la beca, tú no sabes lo que fue aquello [suspira y sonríe] (4-COL1-UCSUR, 2017).
\end{abstract}

Un elemento de atracción para venir a México fue la gratuidad de los estudios de licenciatura, motivación que también se ha identificado en otros estudios a nivel regional, por ejemplo en el caso del estudiantado chileno y colombiano que llega a Argentina a realizar sus estudios de nivel superior (SOSA, 2016). Otra razón fue la posibilidad de obtener una beca para continuar con sus estudios de posgrado, tal y como se señaló en los dos relatos anteriores, pero también hubo factores como los conflictos políticos, económicos y de violencia social que expulsaron a las académicas de sus países de origen. Así se hizo constar en el caso de Panamá, como resultado de la invasión del comando militar estadounidense que dirigió la "Operación Causa Justa", según el discurso del gobierno de ese país, para capturar 
al entonces dictador Manuel Antonio Noriega, acusado de narcotráfico. En el caso de Colombia, por la guerra que el gobierno impulsó contra el narcotráfico que inició desde los ochenta pero que se recrudeció una década después, convulsionando tanto económica como socialmente al país y a la ciudadanía:

\begin{abstract}
Vine a estudiar la licenciatura porque en el tiempo que yo salí de la preparatoria, a finales del 87', había problemas muy fuertes en Panamá de corte político y las universidades estaban cerradas entonces, yo me vine acá. Mis padres me apoyaron y por los problemas políticos que había en el país, la universidad estaba cerrada, no había otra forma de estudiar y no se podía, tuve que elegir en quedarme allá sin estudiar o venirme a estudiar y elegí venirme (1-PAN-UNOR, 2017).
\end{abstract}

Había crisis en los ochenta, cuando yo salgo de mi país había una crisis política y económica muy fuerte en el sur entonces, no se miraba para allá como para que tu dijeras "me voy a ir a Argentina, a Chile a estudiar", el sur no era opción, se miraba a otros lados [...]. Antes de salir de Colombia yo había presentado examen a la universidad y había quedado, pero no podía entrar porque había un paro, todo el tiempo había paros porque la universidad estaba muy politizada, era la época de los ochenta, había mucha susceptibilidad con los movimientos sociales y todo el tema del narcotráfico (2-COL-UCSUR, 2017).

La movilidad académica internacional, en el caso del estudiantado, puede ser entendida de manera general como un proceso en el cual las y los jóvenes tienen la posibilidad de ampliar sus conocimientos y obtener una formación académica en un país distinto al de origen, esa estancia puede ser corta o larga, pero tiene la característica de ser temporal y, en principio, se espera que las y los estudiantes retornen a sus países de origen. En casos de estudio que ya han sido documentados (GÉRARD, et al., 2009; FITTIPALDI et al, 2012; TREJO y SIERRA; 2014) a la movilidad estudiantil internacional se le suele estudiar como el primer paso de un camino que puede terminar como migración internacional. Para el caso que nos ocupa, ese fue un proceso que se dio en el transcurso de la vida estudiantil de algunas de las académicas porque en la toma de decisión para salir de sus países de origen, no tenían pensado quedarse de manera definitiva en México.

En otro estudio (SÁNCHEZ, 2010), se señala que los contextos de salida de las y los migrantes son una forma de conocer las maneras en que serán sus llegadas e inserción a los países de "destino", cuando las personas que migran cuentan, desde su salida, con redes sociales o familiares de apoyo que les esperan y les acogen en los países de "destino", se documenta que su ingreso e inserción en las nuevas sociedades es menos tensionante. Peña (2015) menciona que no es lo mismo las migraciones que se realizan sin documentos que las que se llevan a cabo con el respaldo institucional, como puede ser el trámite de permisos laborales en las oficinas de gobiernos entre los países o de instituciones a través de las cuales se ofrecen y apoyan las estancias laborales o académicas. Por ejemplo, en el caso de algunas de las investigadoras en el estudio, ya habían sido contratadas desde sus países de origen. En el caso de las profesoras que primeramente tuvieron una movilidad académica, ya tenían permisos institucionales, becas en proceso o concedidas, familias o redes de amistades que las esperaban o pares de su mismo país con quienes vivenciaron el desarrollo de un posgrado. Los elementos anteriores hacen suponer que las académicas tuvieron una llegada al país que les facilitó la incorporación a los nuevos espacios sin embargo, son las propias académicas 
quienes brindaron matices y contrastes con respecto a dicho supuesto, no solo con las redes, los nuevos contextos y cultura académica a donde se incorporaron, también con las propias instituciones gubernamentales y las acciones de política migratoria del gobierno mexicano que gestiona el proceso de su ingreso y estancia en el país:

\begin{abstract}
En ese proceso me di cuenta que había otra chica colombiana que venía a estudiar el mismo doctorado que yo, mi director había aceptado en total a 3. Con una de ellas, me coordiné para que llegáramos juntas y tuvimos la experiencia juntas, en el mismo laboratorio, ella no logró terminar, pero ella fue mi apoyo mucho tiempo y entonces no estábamos tan solas nos acogimos las dos [...]. Yo venía de un lugar con muchas reglas, estaba muy formada en un centro donde tenían muchas normas, yo trabajé en laboratorios que tenían seguridad, tú entrabas con tu batita y todo estaba limpio, con códigos diferentes, todo organizadito y todo limpiecito, yo venía de esa formación y cuando llegué dije "!Dios mío! ¿Qué es esto?”. Cuando entro, lo primero que veo en el laboratorio es una mitocondria de unicel volando, le estaba tirando un estudiante a otro, estaban jugando en el laboratorio, era un desorden. Entonces dije "esto es un caos, ¿cómo es posible que tengan esto así?". Yo decía lo que pensaba y por eso en un principio sentí rechazo. Al llegar yo no sabía los códigos mexicanos, las reglas no escritas, eso de "doctor", yo le hablaba de tú a mi director de tesis. [...] Me pregunté ¿Por qué nadie me quiere, por qué me odian y por qué me están mirando así? Estaba siendo muy agresiva con ellos, yo venía de una formación donde decías lo que pensabas pero aquí parece que no está bien visto, [...]. Recuerdo que la primera vez que tuvimos el seminario del grupo, inmediatamente dije "están errados, esto se hace así y lo otro se hace así, es fácil, es pan comido", fui muy directa, agresiva, después lo comprendí, me cayó el veinte (5COL2-UCSUR, 2017).
\end{abstract}

Tengo otra historia que no es tan bonita, que justamente tiene que ver con la parte migratoria, por la que he sufrido muchas penurias porque el sistema migratorio mexicano, en mi experiencia personal, es terrible, ha sido siempre un sufrimiento cada vez que yo tengo que hacer mis actualizaciones, he vuelto llorando a mi casa, tú tienes que tener mucha persistencia y ser muy fuerte porque realmente te abate. Yo en este momento voy a cumplir 17 años en el país, tu pensarás que ya soy mexicana, pues no, por unas equivocaciones de papeles y de procesos me quitaron todos mis años viviendo aquí y estoy como una recién llegada, como "residente temporal", como si fuera recién llegada, me quitaron todo, en el papel me quitaron mis 17 años de vida aquí, borraron mi vida aquí; en lo personal no me lo quitaron, yo tengo esa experiencia vivida, pero me siento devastada, un país al que yo le he brindado todo en estos 17 años, las leyes migratorias y los procesos y trámites que solo ellos entienden, me quitaron todo ese tiempo en el papel y eso también repercute en mis derechos a los que yo debería tener acceso por todos esos años viviendo aquí (3-ARG-UCSUR, 2017).

Lo anterior coincidió con el estudio de Peña (2015) quien documentó la relación del personal mexicano calificado y la tensión existente con el control/apertura del mercado laboral en Alemania, señalando que las personas participantes en su estudio fueron "incluidos por su estatus documentado y por realizar actividades laborales registradas o formales; pero muchos también son excluidos ya que ese mismo estatus legal impone restricciones en sus actividades laborales y en el acceso a los beneficios sociales del estado alemán" (p. 135). En el caso de la investigadora que nosotras señalamos en una de las citas que se expusieron antes, no solo fue excluida sino que las autoridades mexicanas migratorias, en un acto arbitrario, "borraron" a través de una acción institucional, las atribuciones que por derecho le corresponden a la investigadora, al contar con 17 años viviendo y trabajando en el país. 
Después de los estudios de posgrado y del proceso de ingreso al ámbito laboral en México, las académicas entrevistadas experimentaron discriminación durante la transición de una etapa a otra, que en um par de casos no tuvo que ver con su condición de extranjeras, pero sí por ser mujeres en el trabajo que desempeñaban como académicas y por los diferentes grupos académicos de oposición a los que pertenecían, además de que refirieron las experiencias que vivieron en la investigación por no tener la categoría laboral solicitada para ser responsables de proyectos de investigación y para poder publicar sus artículos como primeras autoras, cuando los trabajos los habían iniciado, desarrollado y escrito, ellas:

Empezó ahí un golpeteo y sí fue una cuestión de genero porque con los hombres no se metían, se metían conmigo porque era la única mujer. Se generó una tensión entre los PTC [Profesores de Tiempo Completo] nuevos y los PTC viejos, los PTC nuevos decían que nosotros no los dejábamos hacer cosas nuevas, que estábamos anclados en lo viejo y que lo que los nuevos PTC traían era mejor y no ¡No es que fuera mejor! Es que ellos querían desconocer lo que nosotros habíamos hecho, nuestro trabajo. La bronca era entre los viejos y los nuevos profesores, pero después vino un ataque personal, un ataque de género muy fuerte, por ser mujer, una cuestión de envidia no sé de qué, de mujeres, fíjate, mujeres estudiando género y ellas ahora iniciando el golpeteo hacia mi persona (2-COL-UCSUR, 2017).

Fui y soy profesora de asignatura definitiva de la UNAM. Pero en aquel momento de mi vida eso no era suficiente para la vida y entonces trabajé en una universidad privada, coordinando la elaboración de libros didácticos. Ahí estuve un tiempo, pero ya desde ese momento yo empecé a trabajar mi proyecto de investigación propio. Pero como no pertenecía a ninguna institución como profesora de tiempo completo pues nadie me financiaba nada, pero eso sí, hasta mis proyectos, mis propias ideas en artículos tenían que estar a nombre de otras personas porque ellas sí tenían la titularidad. Solamente hasta que llego aquí, me gano la plaza [de Profesora de Tiempo Completo], entonces sí tengo la posibilidad de meterlo a mi nombre y afortunadamente me gané el financiamiento para desarrollar mi proyecto y ahora mis publicaciones ya solo salen a mi nombre (1-CUB-UCSUR, 2017).

En otros relatos, sí se reconocieron elementos en los que se infiere tensión en el proceso de la inserción laboral, en donde el tratamiento discriminatorio hacia ellas fue puesto en evidencia en los espacios académicos mexicanos, en los siguientes relatos se apreció discriminación hacia las investigadoras por su condición de extranjeras en ambas universidades:

Sí fueron difíciles, especialmente fueron difíciles los primeros cuatro años porque trabajaba por horas. Después empecé poco a poco, concursé en una primera convocatoria para obtener un Tiempo Completo, no tuve suerte y tuve que esperar a una segunda, fue tan difícil la cuestión laboral para incorporarme a la universidad, todo, a las costumbres, a las formas y luego te ven sospechosa porque eres extranjera, a veces hasta te llegas a sentir así, así como sospechosa pero, ¿Sospechosa de qué? Si no hice nada (ríe) (2-COL-UNOR, 2017).

El color de mi piel me delata, pienso "sí, claro, aquí puedes ser morena, pero no negra" (5-COL2-UCSUR, 2017).

Cuando llegas siempre hay un rechazo, hay estereotipos muy marcados que te hacen sentir que tú no eres de aquí, se manejan estereotipos en el sentido de rechazo, eso nos pasa a nosotras las centroamericanas, aquí nos han hecho una fama negativa, eso como extranjera te crea situaciones incomodas, la gente te juzga sin conocerte y muchas veces la gente te juzga con desdén (1-PAN-UNOR, 2017).

v.6

$1-20$

$\mathrm{e} 020021$


En este estudio, se encontró que en varios de los casos, la condición de extranjera en México profundizó la discriminación de las mujeres académicas, especialmente lo vivenciaron así aquellas académicas que se identificaron como afrocolombianas, quienes han construido esa pertenencia étnica "como parte de sus reivindicaciones y, por tanto, de su construcción identitaria” (LAMUS, 2009, p. 110) y también en el caso de las académicas centroamericanas, identidades que siguen siendo invisibilizadas en los espacios de investigación y docencia en México.

Esas tensiones estuvieron mediadas por la experiencia, por la práctica, por el conocimiento y vivencia en las culturas académicas donde se insertaron laboralmente y, con el paso del tiempo, las participantes en el estudio fueron adquiriendo una serie de aprendizajes que les permitió poner en práctica estrategias o acciones para superar situaciones de tensión. En los siguientes relatos, las participantes en el estudio, nos narran como fueron construyendo y reconstruyendo estrategias, así como las maneras de implementarlas en su vida académica cotidiana tanto en la universidad del noreste como en la universidad del centro-sur:

Casi nadie sabe que yo soy extranjera, ya no hablo con acento, porque te digo que desde que llegué me criticaban, me corregían "no digas eso, esto se dice así, aquí está mal visto lo que dices" entonces, cambié, al principio hablaba poco, luego ya aprendí a no tener casi acento. No sé si fue porque, te digo, fue una cosa de mimetizarme para que no me identificaran y no me excluyeran, eso lo fui practicando poco a poco y ahora ya no tengo acento. (2-COL-UCSUR, 2017).

\begin{abstract}
Hay que acoplarse a la forma de vida, respetar las costumbres, tradiciones, sí, pero también hay que marcar la diferencia, tratar de mediar esas dos cosas, ser creativa para resolver los problemas, pero eso lo aprendes con el tiempo porque al principio, en esos primeros años a mi llegada tuve muchos conflictos con todo mundo (ríe). [...]. Me negué a firmar una petición de unas señoras católicas de la colonia en relación a la cuestión del aborto, en aquel entonces teníamos la visita del Papa. [...]. Hoy la gente ha cambiado un poco su mentalidad, la gente intenta ser un poco más abierta dentro y fuera de la academia, pero sigo pensando que México es todavía una sociedad conservadora y eso también se refleja en la universidad. Yo llevo la asignatura Historia de las Mujeres I y II y todavía estos temas hay que discutirlos de forma delicada, con mucho tacto, por lo menos las primeras tres clases, ahora ya aprendí, en las primeras clases me voy con mucho cuidado, luego voy directa, no es que me desvíe de mi camino, simplemente aprendí a ir lento pero eficaz (2-COLUNOR, 2017).
\end{abstract}

Las académicas pusieron en marcha estrategias que ellas mismas fueron aprendiendo, a través de prueba y error, con y frente a los otros. Identificamos, por ejemplo, la estrategia de "mimetización" para no ser excluidas, en este caso, desdibujar el acento del idioma para pasar desapercibida como extranjera, pero aquí cuestionamos si fue una estrategia para accionar su agencia o más bien fue una respuesta a la presión a cierta cultura asimilacionista en México que apunta a homogeneizar lo diferente. Otra estrategia fue la perseverancia y tuvo que ver con el aprendizaje de "ser creativa para resolver los problemas" y las vicisitudes que se les presentaron particularmente en la vida académica, por ejemplo para introducir con "mucho cuidado" una asignatura docente que puede causar tensión en el estudiantado que pertenece a 
una universidad y estado de la República conservador. "Lento pero eficaz" fueron las palabras de otra investigadora para nombrar el aprendizaje que ella obtuvo en su práctica docente, utilizando acciones pragmáticas para alcanzar sus objetivos. Otros tipos de aprendizajes fueron refreídos como "me cayó el veinte", para representar el sentido de la comprensión de lo diverso y el trabajo conjunto con sus pares y colegas en una universidad mexicana.

Con todas esas experiencias las académicas se posicionaron de una manera creativa, pragmática, perseverante y eficaz en los espacios de investigación y docencia, pese a las diferencias de los contextos específicos de cada estado y del nivel de desarrollo en las universidades públicas, desde donde hoy se adscriben. Lo anterior también lo podemos observar en los siguientes relatos donde nos muestran su posicionamiento, esfuerzo y trabajo que han desarrollando a través de los años, en sus diferentes espacios académicos:

Cuando llegué aquí, no había grupos de investigación, yo llegué a picar piedra, no había nada, la tarea de investigador no estaba valorada. Empecé a trabajar casi en solitario, poco a poco y al fin del camino, cuando veo más allá, digo "¡Todo lo que he hecho!". Entonces, sí es reconfortante ver un laboratorio completo funcionando y con alumnos brillantes haciendo investigación, trabajando con ellos, formándolos desde cero, es reconfortante (4-ARG-UNOR).

¿Cuál diría yo que es mi fuerte en la formación del estudiante? Yo me he propuesto tratar de incrementar la formación de investigadores. Desarrollo talleres de tesis y ahí empiezo a formar investigadores. Yo aplico el modelo de argumentación de Toulmin en la eficiencia de titulación. Acabo de escribir un artículo sobre eso. Mi colega con la que estoy trabajando, me dice "tú le estás dando un poder al modelo que no tiene". Yo traté de constatar que empíricamente el modelo funciona para titularse. Ella dice "el modelo en sí mismo no es efectivo, eres tú, el mediador que está haciendo que los chicos se titulen". Algo he de estar haciendo bien en la formación de jóvenes investigadores [sonríe] (1-CUB-UCSUR, 2017).

Me da una gran satisfacción que somos la primera facultad en motivar, desde la infancia, a que niñas y niños estudien ciencias exactas. Inicié ese programa desde el 2003. Tengo alumnos que fueron influenciados por ese programa científico infantil. [...]. Otro proyecto es el de Mujeres en la Ciencia, nació en el 2013 [...], ese año atendimos a 1200 estudiantes de preparatoria, ese proyecto lo que busca es cambiar la perspectiva de vida de las jóvenes, que ellas vislumbren que hay un mundo mucho más allá de la preparatoria y que vean que si lo desean, pueden estudiar una carrera científica (1-PAN-UNOR, 2017).

Si bien a las académicas se les preguntó por el desarrollo de su investigación, la mayoría de ellas, centraron sus relatos en la formación de jóvenes investigadores/as. Lo anterior no significa que las entrevistadas no estén realizando trabajo de investigación, tal y como se puede constatar en la primera tabla que presentamos, ocho de las diez entrevistadas son reconocidas en el Sistema Nacional de Investigadores, una de ellas, (4-arg-unor), en el nivel 3, el máximo nivel. Nosotras pensamos que esa focalización podría estar relacionada con un compromiso y responsabilidad social que las académicas tienen con la sociedad. En México, las académicas no sólo realizan investigación y difunden conocimiento, una de las contribuciones más tangibles que se apreció en sus relatos, fue su quehacer docente, cuya piedra angular es la formación del estudiantado en la ciencia. 


\section{Consideraciones Finales}

Desde las décadas de los ochenta, pero principalmente en los noventa y dos mil, una nueva oleada de migrantes llegó a México conformada por grupos provenientes de varios países del mundo. La diferencia con las décadas anteriores fue que ya no solamente se trató de exilios, también fue por migraciones económicas, por razón de estudio y trabajo, dicha heterogeneidad y diversidad la pudimos advertir en los casos que presentamos en este artículo.

Los motivos que las entrevistadas brindaron para su movilidad y migración fueron por las crisis económicas, por la falta de empleo y por los conflictos sociales y políticos en los que estuvieron inmersos sus países. En varios casos, las razones fueron por el deseo de continuar con su formación académica y profesional. En el estudio, se identificaron algunos elementos que pudieron influir en sus experiencias de migración por ejemplo, la obtención de una beca, la existencia de redes familiares y de apoyo de amistades, los motivos y recursos institucionales y económicos que tuvieron (o no) al momento de la salida de sus países de origen. En ese proceso, se visibilizó como elementos de tensión la condición de académica extranjera en México y la discriminación que experimentaron, especialmente quienes se nombraron así mismas como afrocolombianas y centroamericanas. Algunas de las estrategias que pusieron en marcha para tratar de resolver dichas tensiones fueron la mimetización, la creatividad, el pragmatismo y la perseverancia, pese a las diferencias en el desarrollo socioeconómico de los estados y del desarrollo en la investigación en universidades públicas en donde laboran y de su condición de género por los estereótipos de estigmatización por ser mujeres. Pero esas estrategias no deberían quedar en el plano individual, conocer las experiencias del proceso migratorio de las académicas sudamericanas en México (o de cualquier otro origen), permitiría al gobierno mexicano diseñar e implementar políticas públicas en materia de inmigración para la atracción, incorporación y desarrollo del profesorado universitario que desee trabajar y vivir en el país.

Finalmente, queremos señalar que ya sea que la movilidad académica lleve a las y los jóvenes a migrar de manera permanente o no, una cuestión que en este estudio quedó pendiente de explorar en el futuro, es la direccionalidad de esas movilidades y los significados e impactos en la reconfiguración de las migraciones internacionales calificadas. La llamada direccionalidad Sur-Norte de las migraciones calificadas continúa teniendo como destino principal los espacios académicos estadounidenses sin embargo, coincidimos con Pedone e Izquierdo (2018) al referir que desde finales de los noventa y claramente dos décadas después, existe una reconfiguración de las migraciones en el Sur global en momentos específicos y coyunturas históricas internacionales particulares, que tienen como marco central el desarrollo/ausencia de políticas públicas en dicha materia por parte de los gobiernos. 
Con el estudio que presentamos, podemos afirmar que el Sur también se mueve hacia el Sur, pero hacen falta más estudios en nuestros países sudamericanos, latinoamericanos y del caribe en donde se documenten y analicen ese tipo de flujos y movimentos, poniendo en el centro de los reflectores la participación de las académicas, así como las repercusiones que ese tipo de flujos tiene para la región, de manera que se puedan intuir otras formas de acercarnos al estudio de las migraciones calificadas para comprender esos procesos desde las universidades públicas en el Sur global.

\section{Referencias}

ARIZA, Marina. Ya no soy la que dejé atrás... Mujeres migrantes en República Dominicana. México: IIS-UNAM, Plaza y Valdés Editores, 2000. 300 p. ISBN: 9688568376.

BERTAUX, Daniel. La perspectiva biográfica: validez metodológica y potencialidades. In José Miguel Marinas y Cristina Santamarina (Ed.). La historia Oral: métodos y experiencias. España: Editorial Debate, 1993.p. 149-171. ISBN: 8474447232.

CASTAÑOS, Heriberta. Oleadas de migrantes científicos a México: una visión general. México: UNAM-IIE, 2011. 290 p. ISBN: 9786070222122.

CASTLES, Stephen. y MILLER Mark. La era de la migración: movimientos internacionales en el mundo moderno. México: UAZ, INM, Porrúa, 1993. 388 p. ISBN: 9707015411.

\section{CONSEJO NACIONAL DE CIENCIA Y TECNOLOGÍA. Sistema Nacional de} Investigadores. México: CONACyT. Disponible en: https://www.conacyt.gob.mx/index.php/el-conacyt/sistema-nacional-de-investigadores. Consultado en: 26 abril 2019.

DE VILLOTA, Paloma. Globalización y género. Madrid: Síntesis, 1999. 416 p. ISBN: 9788477386469.

DELGADO, Raúl. Dos líneas de investigación de frontera. In: DELGADO, Raúl (Coord.). Hacia dónde va la ciencia en México. México: CONACyT/AMC, 2014. p. 135-148. ISBN: 9786078273041.

DUBAR, Claude. La crisis de las identidades. La interpretación de una mutación. Barcelona: Balleterra, 2002. 280 p. ISBN: 8472901858.

ESTRADA, Olga; IZQUIERDO, Isabel. Hacedoras de vocês: Seis estudios sobre mujeres, género y feminismos en México. Monterrey: UANL, 2011. ISBN: 9786074336801.

ESTRADA, Olga. Vivencias, realidades y utopías: México. Las causas civiles, sociales y políticas de las mujeres en México. Siglo XX y un estudio histórico de las mujeres en Nuevo León (1980-2010). Monterrey: UANL, 2012. 477 p. ISBN: 9786074337983. 
FITTIPALDI, Rosa; MIRA, Silvia; ESPASA, Lorena. Movilidad de estudiantes de educación superior en el contexto de las migraciones contemporáneas: La experiencia de la Universidad Nacional del Sur. Revista Universitaria de Geografía. n. 21, p. 113-136, 2012.

FLORES, Glenda. Racialized Tokens: Latina Teachers Negotiating, Surviving and Thriving in a White Woman's Profession. Qualitative Sociology. n. 34, p. 313-335, 2011.

GÉRARD, Etienne; MALDONADO, Alma. Polos de saber y cadenas de saber: un análisis del impacto del proceso de movilidad académica entre México y el extranjero. Revista de la Educación Superior, n. 38, p. 49-62, 2009.

GREGORIO, Carmen. Migración femenina. Su impacto en las relaciones de género. Madrid, Narcea, 1998. 288 p. ISBN: 9788427712508.

HOPENHAYM, Fernanda. Las mujeres en los sistemas científicos latinoamericanos: la otra fuga de cerebros. 2009. Tesis (Maestría en Estudios Latinoamericanos) - Facultad de Filosofía y Letras, Universidad Nacional Autónoma de México, 2009.

IREDALE, Robyn. Gender, Immigration Policies and Accreditation: Valuing the Skills of Professional Women Migrants. Geoforum. n. 36, p. 155-166, 2005.

IZQUIERDO, Isabel. Las científicas y los científicos extranjeros que llegaron a México a través del Subprograma de Cátedras Patrimoniales del CONACyT. Revista de la Educación Superior. n. 155, p. 61-79, 2010.

IZQUIERDO, Isabel. Las científicas inmigrantes en México. In: ESTRADA, Olga Nelly; IZQUIERDO, Isabel (Coord.). Hacedoras de voces: seis estudios sobre mujeres, género y feminismo en México. Monterrey: UANL, 2011, p. 19-34. ISBN: 9786074336801.

IZQUIERDO, Isabel; GUZMÁN, Gezabel. Identidad performativa de académicas latinoamericanas en México: ser altamente calificada y la posibilidad de la maternidad presente. In: IZQUIERDO, Isabel (Coord.). Identidades en movimiento. Inmigrantes en el México contemporáneo. Morelos: UAEM/FONTAMARA, 2016, pp. 115-132. ISBN: 9786077363576.

KASTERSZTEIN, Joseph. Les stratégies identitaries des acteurs sociaux: aproche dynamique des finalités. In: CAMILLERI, Carmel et al. Stratégies identitaires. París: Presses Universitaires de France, p. 27-41, 1999. ISBN: 9782130428589.

KOFMAN, Eleonore; RAGHURAM, Parvati. Gender and Skilled Migrants: into and Beyond the Work Place. Geoforum. n. 36, p. 149-154, 2005.

LAMUS, Doris. Mujeres negras/afrocolombianas en los procesos organizativos en Colombia. Un aporte al estado del debate. Reflexión Política. n. 21, p. 108-125, 2009.

LARROSA, Jorge. Sobre la experiencia. Separata Revista Educación y Pedagogía. v. 18, p. 43-51, 2006. Disponible en:

http://aprendeenlinea.udea.edu.co/revistas/index.php/revistaeyp/article/view/19065/16286 . Consultado en: 14 nov. 2017. 
LIPSZYC, Cecilia. Feminización de las migraciones: sueños y realidades de las mujeres migrantes en cuatro países de América Latina. In: CONGRESO CAMINAR SIN MIEDOS, 2005, Montevideo: URBAL Red12MujerCiudad. p. 3-15.

MARTÍNEZ-ASSAD, Carlos. De extranjeros e inmigrantes en México. México: UNAM, 2008. 598 p. ISBN: 9789703251438.

MENDOZA, Cristóbal; STANISCIA, Barbara; ORTIZ, Ana. Migración y movilidad de las personas calificadas: nuevos enfoques teóricos, territorios y actores. Revista Bibliografica de Geografía y Ciencias Sociales. V. 21, n. 1, p. 1-22, 2016. Disponible en:

http://www.ub.edu/geocrit/b3w-1166.pdf . Consultado en: 18 dic. 2018.

MEYER, Eugenia; SALGADO Eva. Un refugio en la memoria. La experiencia de los exilios latinoamericanos en México. México: UNAM, OCÉANO, 2002. 361 p. ISBN: 9706516883 .

OREGIONI, María. La internacionalización universitaria desde una perspectiva situada: tensiones y desafíos para la región latinoamericana. Revista Internacional de Educação Superior, v. 3, n. 1, p. 114-133, 2017.

ORGANIZACIÓN DE LAS NACIONES UNIDAS. Estudio mundial sobre el papel de la mujer en el desarrollo, 2004. La mujer y la migración internacional. Nueva York: ONU, 2006. 97 p. ISBN: 9213301952.

ORTIZ, Ana; MENDOZA, Cristóbal. Mujeres expatriadas en México: trabajo, hogar y vida cotidiana. Migraciones Internacionales. n. 4, p. 1- 32, 2007.

PARELLA, Sonia. Mujer, inmigrante y trabajadora: la triple discriminación. Barcelona: Anthropos, 2003. 413 p. ISBN: 8476586698.

PEDONE, Claudia; IZQUIERDO, Isabel. Programas de atracción de académicos. Los casos del Subprograma de Cátedras Patrimoniales en México y del Programa Prometeo en Ecuador. Education, Policy Analysis Archive, v. 26, n. 10, p. 1-16, 2018. Disponible en: https://epaa.asu.edu/ojs/article/view/3425 . Consultado en: 18 dic. 2018.

PEÑA, Jesús. Saber alemán: inserción de migración calificada mexicana en el mercado laboral de Berlín. Revista Internacional de Estudios Migratorios. n. 5, p. 119-148, 2015.

RIAÑO, Yvonne. Migration de femmes latino-américaines qualifiées universitaires en Suisse: Géographies migratoires, motifs de migration et questions de genre. In: BOLZMAN, C.; CARBAJAL, Myrian; MAINARDI, Giuditta (Ed.). La Suisse au rythme latino: Dynamiques migratoires des Latino-américains: logiques d'action, vie quotidienne, pistes d'intervention sociales et de santé. Geneve: Editions IES, p. 115-136, 2007. ISBN: 9782882240781 .

RIAÑO, Yvonne; BAGHDADI, Nadia. Understanding the Labour Market Participation of Skilled Immigrant Women in Switzerland: The Interplay of Class, Ethnicity, and Gender. International Migration \& Integration. n. 8, p. 163-183, 2007. 
ROMO, Rosa. Argentinos en México. Procesos de exilio e innovación universitaria. Revista Iberoamericana para la Investigación y el Desarrollo Educativo, n. 10, p. 1-12, 2015.

SÁNCHEZ, Adolfo. Recuerdos y reflexiones del exilio. Barcelona: Associació d'Idees, GEXEL, 1997. 190 p. ISBN: 9788487478192.

SÁNCHEZ, Antonia. Las redes migratorias y la intervención social: un estudio de caso de la comunidad ucraniana en la región de Murcia. Cuadernos de Trabajo Social, n. 23, p. 65-83, 2010.

SKACHKOVA, Penka. Academic Careers of Immigrant Women Professors in the U.S. Higher Education. The International Journal of Higher Education and Educational Planning, n. 53, p. 697-738, 2007.

STANG, María. Saberes de otro género Emigración calificada y relaciones intergenéricas en mujeres argentinas y chilenas. Informe Final del concurso Migraciones y modelos de desarrollo en América Latina y el Caribe. Programa Regional de Becas CLACSO, 2006.

Disponible en: http://bibliotecavirtual.clacso.org.ar/ar/libros/becas/2005/2005/migra/stang.pdf Consultado en: 9 oct. 2018.

TREJO, Alma; SIERRA, Carlos. Gestión de la movilidad estudiantil en Estados Unidos. Dimensionando a los estudiantes colombianos y mexicanos. Revista Camino real: estudios de las hispanidades norteamericanas. n. 9, p. 119-141, 2014.

VALLES, Miguel. Entrevistas cualitativas. Cuadernos metodológicos. Madrid: Centro de Investigaciones Sociológicas, 2002. 195 p. ISBN: 847476-3428.

WOO, Ofelia. La invisibilidad en el proceso migratorio: las mujeres migrantes. Frontera Norte. n. 7, p. 139-148, 1995. 